\title{
Erratum: Beam emittance measurement with laser wire scanners in the International Linear Collider beam delivery system [Phys. Rev. ST Accel. Beams 10, 112801 (2007)]
}

\author{
I. Agapov, G. A. Blair, and M. Woodley \\ (Received 14 January 2008; published 23 January 2008)
}

The email address of one of the authors (Mark Woodley) was given incorrectly: instead of mwd@slac.stanford.edu one should readmdw@slac.stanford.edu.

There is a typographical error on page 3 . The projected emittance definition should read

$$
\epsilon_{y}^{2}=\operatorname{det}\left[\begin{array}{cc}
\left\langle y^{2}\right\rangle & \left\langle y y^{\prime}\right\rangle \\
\left\langle y y^{\prime}\right\rangle & \left\langle y^{\prime 2}\right\rangle
\end{array}\right] .
$$

Also on page 3 , there is a typographical error in the definition of $M_{X Y}$. It should read

$$
M_{X Y}=\left[\begin{array}{cccc}
R_{11,1} R_{33,1} & R_{11,1} R_{34,1} & R_{12,1} R_{33,1} & R_{12,1} R_{34,1} \\
R_{11,2} R_{33,2} & R_{11,2} R_{34,2} & R_{12,2} R_{33,2} & R_{12,2} R_{34,2} \\
R_{11,3} R_{33,3} & R_{11,3} R_{34,3} & R_{12,3} R_{33,3} & R_{12,3} R_{34,3} \\
\cdots & \cdots & \cdots & \cdots
\end{array}\right] .
$$

\section{ACKNOWLEDGMENTS}

We would also like to acknowledge the support of the U.S. Department of Energy, Contract No. DE-AC02-76SF00515. 